ÉGYPTE monde arabe

\section{Égypte/Monde arabe}

$12 \mid 2015$

Evolution des systèmes médiatiques après les révoltes arabes

\title{
Contestation en ligne dans la Tunisie prérévolutionnaire. L'expérience de SfaxOnline
}

Michele Carboni, Maria Paola Crisponi et Giovanni Sistu

\section{(2) OpenEdition}

\section{Journals}

Édition électronique

URL : https://journals.openedition.org/ema/3463

DOI : $10.4000 /$ ema.3463

ISSN : 2090-7273

Éditeur

CEDEJ - Centre d'études et de documentation économiques juridiques et sociales

Édition imprimée

Date de publication : 25 mars 2015

Pagination : 231-252

ISBN : 9782905838858

ISSN : 1110-5097

Référence électronique

Michele Carboni, Maria Paola Crisponi et Giovanni Sistu, « Contestation en ligne dans la Tunisie prérévolutionnaire. L'expérience de SfaxOnline », Égypte/Monde arabe [En ligne], 12 | 2015, mis en ligne le 25 mars 2015, consulté le 07 juillet 2022. URL : http://journals.openedition.org/ema/3463 ; DOI : https://doi.org/10.4000/ema.3463 


\section{Michele Carboni, Maria Paola Crisponi \& Giovanni Sistu}

\section{RÉSUMÉ / ABSTRACT}

Au début des années 90, la Tunisie devient le premier pays arabe et africain à se connecter à Internet, faisant de la diffusion du réseau en ligne un motif d'orgueil et un instrument de propagande. Le régime de Ben Ali investit avec conviction dans les nouvelles technologies en agissant simultanément sur deux fronts. Si d'un côté, des politiques visant à leur promotion sont adoptées, de l'autre, un système de contrôle rigide se met en place. Le régime tunisien deviendra rapidement l'un des plus répressifs au monde en ce qui concerne le contrôle de la toile. Malgré cela, à la fin des années 90, les premiers signes de politisation, utilisée à des fins de contestation, apparaissent sur Internet.

L'expérience du site SfaxOnline, qui apparait en 2008 et donne forme à cette contribution, concourt à l'approfondissement de la connaissance de l'utilisation de la toile à des fins contestataires dans la Tunisie prérévolutionnaire. En effet, ce n'est pas la Révolution qui a transformé Internet en outil de contestation. Le cas de SfaxOnline présenté dans cette recherche témoigne de l'antériorité du phénomène tout en contribuant à faire comprendre comment Internet a réussi à conquérir un espace qui deviendra par la suite fondamental dans les dynamiques révolutionnaires.

L'histoire de SfaxOnline contribue par ailleurs à corriger l'idée - qui ne correspond pas à la réalité - d'un peuple tunisien apathique et totalement passif face au régime dictatorial. Jusqu'à la Révolution de 2011, cette image a caractérisé différentes analyses sur les Tunisiens et sur le monde arabe en général.

MOTS CLÉS :

Tunisie, Internet, Sfax, contestation, cyber activisme, TIC.

At the beginning of the '90s, Tunisia became the first Arab and African country to connect to the Internet. Ben Ali's regime invested heavily in new technologies, working on two fronts. On the one hand, it adopted policies aimed at promoting technological development. On the other hand, it enforced a rigid and articulated system of control. Nevertheless, by the end of the '90s, the first signs of politicization of the Net started emerging. 
The story of the SfaxOnline helps us to gain a deeper knowledge of the use of the Internet for political contestation in pre-revolutionary Tunisia.

\section{KEYWORDS:}

Tunisia, Internet, Sfax, Contestation, Cyberactivism, ICT.

MICHELE CARBONI est actuellement est chercheur associé au CRENoS, Université de Cagliari et Université de Sassari (Italie). II a obtenu son Master en International Cooperation - Development à I'ISPI (Institut pour les Etudes de Politique Internationale, Milan) et son Doctorat en Etudes Africaines à I'Université de Cagliari.

Email :michele.carboni@crenos.unica.it

MARIA PAOLA CRISPONI est actuellement assistante de recherche au CRENoS, Université de Cagliari et Université de Sassari (Italie). Elle a obtenu son Máster Europeo en Estudios Latinoamericanos : Diversidad Cultural y Complejidad Social à la Universidad Autónoma de Madrid.

Email : mariapaola.crisponi@crenos.unica.it

GIOVANNI SISTU est professeur de géographie à I'Université de Cagliari et chercheur au CRENoS, Université de Cagliari et Université de Sassari (Italie).

Email : sistug@unica.it

MICHELE CARBONI is currently Associate Researcher at CRENoS, University of Cagliari and University of Sassari, Italy. He holds a Master in International Cooperation - Development from ISPI (Institute for International Political Studies, Milan), and a PhD in African Studies from the University of Cagliari.

MARIA PAOLA CRISPONI is currently Assistant Researcher at CRENoS, University of Cagliari and University of Sassari, Italy. She holds a Máster Europeo en Estudios Latinoamericanos: Diversidad Cultural y Complejidad Social from the Universidad Autónoma de Madrid.

GIOVANNI SISTU is Professor of Geography at the University of Cagliari and Researcher at CRENoS, University of Cagliari and University of Sassari (Italy). 


\section{REMERCIEMENTS :}

Les auteurs remercient vivement tous les protagonistes de l'expérience de SfaxOnline. Un remerciement particulier à Mohamed Aloulou, Mohamed Ali Ayadi, Ali Elleuch, Raouf Ellouze et Chokri Yaich. Cette recherche a été cofinancée par les fonds de la loi 7/2007 RAS, projet "Il ruolo delle Università e dei centri di ricerca nei processi di democratizzazione dei Paesi arabi mediterranei". 


\section{CONTESTATION EN LIGNE DANS LA TUNISIE PRÉRÉVOLUTIONNAIRE L'EXPÉRIENCE DE SFAXONLINE}

\section{INTRODUCTION}

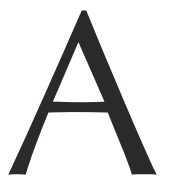

vant la Révolution tunisienne, I'utilisation d'Internet comme espace de contestation et de résistance au régime de Ben Ali était un phénomène peu connu et peu étudié ${ }^{1}$. D'une manière plus générale, avant les printemps arabes, parler de médias dans le monde arabe (et pas seulement en Tunisie) signifiait parler presque exclusivement $\mathrm{d}^{\prime} \mathrm{Al}$ Jazeera $^{2}$. Le fait qu'Internet - et en particulier Facebook - ait joué un rôle dans les dynamiques révolutionnaires tunisiennes est connu et incontestable. Toutefois, il est aussi vrai que ce rôle a été limité ${ }^{3}$. D'une manière plus générale, le rôle des Techniques de l'Information et de la Communication (TIC) peut être qualifié, pour reprendre la synthèse efficace de Béchir Ayari et Geisser ${ }^{4}$, de certes non marginal, mais non déterminant non plus. Ce rôle est ainsi différent de celui que présente les mass médias en particulier, qui font de la toile (et des TIC en général) le deus ex machina de la révolte ${ }^{5}$.

Une emphase excessive à propos du rôle d'Internet dans les dynamiques révolutionnaires risque d'offusquer un autre aspect

1. Lecomte, 2011 ; Mihoub, 2011.

2. Gonzalez-Quijano, 2013.

3. Touati, 2012.

4. Béchir Ayari, Geisser, 2011.

5. Filiu, 2011 ; Kallander, 2013 ; Soussi, 2011 ; Touati, 2012. 
important : ce n'est pas la Révolution qui a fait d'Internet, soudainement, un espace de contestation, de critique, de résistance ou d'opposition ${ }^{6}$. En réalité, les premiers signes de politisation de la toile - et de son utilisation à des fins contestataires - remontent déjà à la fin des années 90, bien avant la Révolution

L'expérience du site SfaxOnline,qui apparait en 2008, contribue en premier lieu à approfondir la connaissance même de l'utilisation de la toile à des fins contestataires dans la Tunisie prérévolutionnaire. Le site est une expression d'opposition de facto, riche de contenus, destiné à faire connaître la gestion dramatique de la ville de Sfax, conséquence directe de l'incapacité des administrations locales ainsi que du manque de planification sérieuse de la part d'un régime plein de contradictions et de distorsions.

Au fil des années, SfaxOnline a servi à informer les habitants de Sfax - l'un des motifs pour lesquels il nait - sur différents sujets liés à la vie de la ville, mais il a aussi servi à mobiliser les citoyens et à les pousser à participer activement à la vie politique. Informer et mobiliser, en invitant à la participation : le site poursuit et rejoint ces deux objectifs, jouant ainsi un rôle similaire (toutes proportions gardées) à celui qui incombera à Internet (et surtout à Facebook) dans les événements révolutionnaires. C'est aussi en cela que SfaxOnline mérite attention ${ }^{8}$. $S^{\prime}$ il est vrai qu'Internet a joué un rôle important dans la Révolution, il est indispensable, pour comprendre comment il a réussi à conquérir cet espace, de comprendre le parcours qui a conduit à la construction de cet espace et de ce rôle.

6. Lecomte, 2011 ; Khamis, 2013 ; Touati, 2012.

7. Gonzalez-Quijano, 2013 ; Soussi, 2011 ; Touati, 2012.

8. Cette recherche naît de la rencontre avec Chokri Yaich, géomorphologue de I'Ecole Nationale d'Ingénieurs de Sfax et, depuis octobre 2011, député à I'Assemblée Nationale Constituante. Yaich a joué un rôle fondamental dans différentes phases de I'histoire de SfaxOnline, aussi bien dans la conception que dans la constitution du groupe de travail, et bien sûr dans l'élaboration des contenus et dans I'animation du site. La rencontre entre Yaich et l'un des auteurs de cet article travaillant alors lui aussi à un projet de développement remonte à 2007.

$C^{\prime}$ est à travers Yaich que les auteurs prennent connaissance du site et commencent à le suivre, dès sa phase initiale. A la suite de la Révolution, dans un climat inédit de liberté d'expression, la décision fut prise d'approfondir les thèmes liés à l'expérience de SfaxOnline. Une série de missions en Tunisie, facilitées par le même Yaich, met alors en contact les auteurs avec d'autres protagonistes du site. 
Cette contribution est fondée principalement sur des données recueillies au cours de dix entretiens réalisés entre 2012 et 2013 en Italie et en Tunisie. Ces entretiens ont concerné cing des protagonistes principaux du site (y compris Yaich), interrogés chacun pendant au moins deux heures.

Chaque interviewé a raconté son expérience personnelle au sein de SfaxOnline, comment il est arrivé à faire partie du groupe de travail et quelle a été sa contribution. Chacun a aussi raconté l'histoire du groupe, comment celui-ci a pris forme, le travail qu'il y a fait et ce dont il s'est occupé, entre 2008 et 2013.

L'analyse se fonde aussi sur les matériaux du site, les posts et les commentaires (ultérieurement commentés et illustrés, dans différents cas, par les mêmes auteurs qui les avaient écrits), mais aussi sur un vaste corpus de conversations informelles et de courriels échangés avec certains animateurs du site, recueillis à partir de 2009. Les échanges avec Chokri Yaich ont été particulièrement fréquents et réguliers.

Le choix des interviewés, supporté et facilité par Yaich, s'est porté sur des habitants de Sfax résidant en ville et non à l'étranger. Sans vouloir minimiser le rôle de ces derniers, les auteurs ont préféré se focaliser sur les animateurs qui ont réussi à être actifs non seulement online mais aussi offline. L'expérience de SfaxOnline n'a en effet pas été une simple aventure numérique. Un groupe restreint d'internautes - dont les interviewés représentent une bonne part - a rapidement commencé à créer une vie associative qui sera aussi analysée dans cette contribution. Le travail de SfaxOnline a été extrêmement collaboratif, dans le sens d'un travail de groupe allant au-delà de la simple somme de contributions personnelles. Pour ce motif, nous avons décidé de reporter des extraits d'entretiens sans indiquer l'identité de l'interviewé, faisant de ce traitement un récit d'une seule voix.

\section{LA POLITISATION DU RÉSEAU : DETAKRIZÀ LA RÉVOLUTION}

En 1991, la Tunisie devient le premier pays arabe et africain à se connecter à Internet ${ }^{9}$. Le régime de Ben Ali investit avec conviction dans les nouvelles technologies, faisant de la diffusion $d^{\prime}$ Internet - et du développement des TIC en général - un motif d'orgueil et un instrument de propagande.

9. Kallander, 2013 ; Touati, 2012. 
Des politiques liées à la diffusion de la toile sont adoptées et des initiatives concrètes sont lancées afin de faciliter les connections privées et publiques ${ }^{10}$. Dans le même temps, un système de contrôle rigide et articulé voit le jour ${ }^{11}$. II s'agit d'un "mariage paradoxal », pour reprendre l'expression de Soussi ${ }^{12}$ : promotion d'Internet et soutien de son développement d'un côté ; contrôle, censure puis répression de l'autre ${ }^{13}$. L'Etat voudrait en effet pouvoir bénéficier des retombées positives en termes économiques du développement d'Internet dans le commerce, dans l'instruction et dans la recherche, mais dans le même temps, il en craint les conséquences au niveau politique et social et travaille constamment à contrôler l'espace virtue $\left.\right|^{14}$. Tout doit passer par I'Agence Tunisienne d'Internet (ATI), opérateur unique jusqu'en 1997. L'ouverture successive à de nouveaux fournisseurs, comme l'ouverture, dans le pays entier, des Publinet (cybercafés), ne dessert pas ce contrôle : tout le monde doit régulièrement fournir des informations sur les internautes tunisiens ${ }^{15}$. Progressivement, le régime adopte des mesures de contrôle de plus en plus sophistiquées. Censures et violations des comptes privés deviennent monnaie courante ${ }^{16}$. De la même manière, les sanctions, les menaces et les intimidations sont constantes, associées à des incarcérations et à des tortures ${ }^{17}$. Un tel climat conduit bientôt les internautes à s'autocensurer. Le régime tunisien deviendra rapidement I'un des plus répressifs dans le contrôle de la toile, au même niveau que des pays tels que l'Iran, la Chine et l'Arabie Saoudite ${ }^{18}$.

\section{Les premiers signes}

Le régime craint qu'Internet ne devienne un instrument pour contourner la censure et pour diffuser librement I'information ${ }^{19}$ dans un pays où les médias traditionnels ont toujours été contrôlés rigoureusement. Ces craintes se révèlent d'ailleurs bien fondées et en effet, comme annoncé ci-dessus, une certaine vocation politique d'Internet commence à émerger dès la fin des années 90.

10. Touati, 2012.

11. Kuebler, 2011 ; Mihoub, 2011.

12. Soussi, 2011.

13. Barhoumi, 2012 ; Kallander, 2013.

14. Lecomte, 2011 ; Kuebler, 2011 ; Touati, 2012.

15. Mihoub, 2011.

16. Barhoumi, 2012.

17. Mihoub, 2011.

18. Kuebler, 2011.

19. Barhoumi, 2012 ; Lecomte, 2011. 
La newsletter Takriz " casse-pieds » à laquelle certains auteurs font remonter la naissance de la cyber dissidence, date de janvier $1998^{20}$. Créée par deux étudiants, elle est rapidement gérée et animée par un groupe de membres permanents dont beaucoup résident à l'étranger. Jusqu'en 2000, lorsqu'elle devient un magazine en ligne, elle dénonce le népotisme, la corruption et le clientélisme de la société tunisienne d'abord puis attaque de manière plus directe le régime de Ben Ali. La politique n'est pas le seul tabou franchi par Takriz: on y parle aussi ouvertement de sexe et de religion. Takriz sera censuré et contraint au silence en 2002 ; il réapparaitra en 2009 sur Facebook et Twitter ainsi que comme magazine en ligne ${ }^{21}$.

En mai 2000, cinq Tunisiens anonymes résidant à l'étranger, probablement des réfugiés politiques islamistes des années $90^{22}$, lancent la liste Tunisnews, qui diffuse quotidiennement des informations politiques importantes (en français et en arabe) non relayées par les médias nationaux, sur les droits de l'homme, les droits civils et l'activité de I'opposition $^{23}$. Tunisnews s'appuie sur un serveur étranger - pratique qui sera successivement extrêmement diffusée - en l'occurrence suédois ${ }^{24}$. En juillet 2001 apparaît TUNeZINE (site et forum), fondé par Ettounsi ("le Tunisien", pseudonyme de Zouhair Yahyaoui) puis animé par différents tunisiens résidant à l'étranger. Fortement orienté sur la satire (comme Takriz), cette expérience se heurte elle aussi rapidement à l'hostilité du régime. L'année suivante, au lancement de TUNeZINE, Ettounsi est emprisonné. Il sera relâché 18 mois plus tard mais mourra d'infarctus à 37 ans en mars 2005, terriblement éprouvé par la détention, la torture et plusieurs grèves de la faim. Il deviendra un symbole de la lutte contre la censure et un véritable martyr de la cyber dissidence ${ }^{25}$.

Au début de l'année 2002 apparaît un autre site déterminant: RéveilTunisien (RT). Géré en grande partie par des non Tunisiens, il nait avec un ton plus sérieux mais $s^{\prime}$ oriente rapidement vers la satire avec une empreinte marquée par le défi au régime ${ }^{26}$. Nawaat apparait en 2004, fondé par deux Tunisiens résidant à l'étrangers - Sami Ben Gharbia et Riadh Guerfali - déjà présents sur le forum TUNeZINE et géré, encore une fois, en grande partie par des Tunisiens de la diaspora $^{27}$. En réaction à RT et à TUNeZINE, Nawaat se présente

20. Lecomte, 2010 ; Mihoub, 2011.

21. Kuebler, 2011 ; Lecomte, 2010 ; Mihoub, 2011.

22. Touati, 2012.

23. Chouikha, 2011.

24. Lecomte, 2010 ; Mihoub, 2011 ; Chouikha, 2011.

25. Ben Mhenni, 2009 ; Soussi, 2011 ; Kallander, 2013 ; Lecomte, 2010.

26. Lecomte, 2010 ; Touati, 2012.

27. Lecomte, 2011. 
comme un produit $100 \%$ tunisien ${ }^{28}$. Le site devient rapidement l'un des portails de référence pour l'information politique ${ }^{29}$ et maintiendra ce rôle longtemps. Nawaat publiera les célèbres cablogrammes de Wikileaks et jouera un rôle de premier ordre durant la Révolution. En 2011, il recevra le prix Net-Citoyen de Reporters sans frontières ${ }^{30}$.

Dans ce que Lecomte ${ }^{31}$ identifie comme la première phase de contestation tunisienne en ligne - et qui va de la fin des années 90 jusqu'à la moitié de la décennie suivante - les "cyber dissidents", souvent définis ainsi par les internautes tunisiens ${ }^{32}$ agissent à travers des sites collectifs, des listes de diffusion et des forums (Takriz, TUNeZINE, RT, Nawaat et Tunisnews représentent des acteurs pionniers). Beaucoup de ces dissidents choisissent l'anonymat, comme beaucoup d'internautes de la diaspora, choix justifié par la crainte de rétorsion pouvant frapper les membres de la famille restés au pays ${ }^{33}$. Dans cette première phase, le rôle des dissidents résidant à l'étranger est fondamental ${ }^{34}$. Il s'agit de Tunisiens qui résident dans des pays où I'accès à Internet est plus facile et plus libre, ce qui les a probablement rendus plus audacieux par rapport aux internautes résidant en Tunisie. Ces premiers cyber dissidents ne font pas partie d'organisations politiques ou militantes, ils sont minoritaires et même isolés par rapport à la majorité des internautes tunisiens et revendiquent leur indépendance dans I'univers de I'opposition tunisienne officielle. Ils en prennent en effet leurs distances puisqu'ils estiment celle-ci liée, voire asservie, au pouvoir, et ils en font souvent un objet de critique satirique ${ }^{35}$.

\section{Des blogs à Facebook}

Au milieu des années 2000, la critique en ligne se déplace progressivement des sites collectifs, des listes de diffusion et des forums vers les blogs, qui dominent la seconde phase de la contestation numérique tunisienne ${ }^{36}$. Leur nombre augmente et surtout, le nombre de contestataires numériques qui choisissent cet espace pour s'exprimer explose. Beaucoup de ces bloggers, qui critiquent le régime tout en refusant l'étiquette d'opposants, de militants ou de dissidents, vivent en

28. Lecomte, 2010.

29. Kuebler, 2011.

30. Chouikha, 2011; Reporters sans frontières, 2011 ; Kuebler, 2011.

31. Lecomte, 2011.

32. Lecomte, 2010.

33. Kuebler, 2011 ; Lecomte, 2010.

34. Chouikha, 2011 ; Mihoub, 2011 ; Lecomte, 2011.

35. Lecomte, $2010 ; 2011$.

36. Lecomte, 2011. 
Tunisie mais interagissent de manière croissante avec les internautes et les bloggers de la diaspora. Dans les blogs, où la satire continue à être une modalité d'expression fréquente, la critique a plusieurs formes: allusions, vignettes, anecdotes et récits d'expériences personnelles. Le phénomène prend des proportions importantes, à tel point que même la presse commence à s'intéresser à la blogosphère ${ }^{37}$.

Les bloggers s'évertuent à contourner la censure d'un régime qui affine lui aussi ses armes et continue d'exercer un contrôle envahissant. Au fil des ans, des sites et des pages web d'opposants, des ONG, des quotidiens étrangers, des sites de partages de photos et de vidéos (Flickr, Youtube et Dailymotion, pour n'en citer que quelques uns) sont rendus inaccessibles. Les sites, les pages web et même les boîtes e-mail d'opposants (ou d'internautes supposés tels), de bloggers et de journalistes professionnels sont régulièrement violés ${ }^{38}$.

Cependant, durant cette phase, les cyber dissidents (qu'ils se définissent ou non ainsi), malgré l'augmentation de leur visibilité, constituent une minorité exiguë dans l'univers numérique tunisien. La majorité des internautes et les bloggers eux-mêmes ne s'occupent pas de politique. Ceux qui le font sont ainsi très critiques par rapport à ce désintérêt apparent, parfois considéré comme une acceptation tacite de l'état des choses et donc du régime. C'est à ce propos qu'en 2006, Sami Ben Gharbia (I'un des fondateurs du Nawaat) qualifiera la blogosphère tunisienne de "lobotomisphère" ${ }^{39}$.

2008 représente un tournant dans l'utilisation d'Internet comme instrument de contestation: c'est le début de la troisième phase dans la périodisation proposée par Lecomte ${ }^{40}$, celle qui correspond à I'affirmation des réseaux sociaux et en particulier de Facebook, qui explose ${ }^{41}$ : de 16000 Tunisiens inscrits au début de l'année, ils deviennent 100000 en septembre de la même année ${ }^{42}$. Facebook devient aussi l'instrument préféré des internautes qui traitent de politique et contestent le régime, même si la politique intérieure demeure un thème rarement approché par la majorité des internautes ${ }^{43}$. 2008 est aussi l'année des révoltes dans le bassin minier de Gafsa ${ }^{44}$. Dans le silence absolu des médias traditionnels, de nombreux témoignages des

37. Lecomte, 2010

38. Barhoumi, 2012 ; Lecomte, 2011 ; Touati, 2012.

39. Lecomte, 2010.

40. Lecomte, 2011.

41. Chouikha, 2011.

42. Touati, 2012.

43. Lecomte, 2010, 2011 ; Kuebler, 2011.

44. Allal, 2010 ; Chouikha, Geisser, 2010. 
répressions violentes, reprises par des téléphones portables, finissent sur Internet ${ }^{45}$. Le régime se rend compte des risques liés au caractère participatif de la toile ainsi que de son potentiel subversif et la répression numérique s'intensifie jusqu'à bloquer Facebook pour quelques jours, du 24 août au 2 septembre $2008^{46}$. Cet arrêt momentané n'empêche en rien la montée du réseau social puisque le nombre d'inscrits passe de 800000 en octobre 2009 à quasiment 2000000 en janvier $2011^{47}$. L'espace numérique tunisien commence à devenir réellement un espace public ${ }^{48}$.

En avril 2010 encore, 200 sites et blogs sont bloqués, aussi bien nationaux qu'étrangers. Par réaction, une journée de manifestation est organisée le 22 mai suivant, "Nhar ala Ammar", "la journée contre Ammar ». C'est en effet ce dernier qui personnifie la censure et prend parfois le surnom de "Mkass", " ciseaux » en tunisien ${ }^{49}$. Il s'agit $d^{\prime}$ une tentative importante de faire descendre la contestation en ligne dans la rue. L'intention des organisateurs n'était pas tant de critiquer le régime que de rappeler l'attention sur les nécessités de la liberté $\mathrm{d}^{\prime}$ expression. La manifestation n'aura finalement pas lieu, entre autres parce que deux bloggers seront arrêtés la veille et contraints à tourner une vidéo dans laquelle ils invitent les manifestants potentiels à se désister ${ }^{50}$.

Le mécontentement monte dans l'ensemble du pays et cette énième tentative de le réprimer ne changera pas le sort d'un régime dont les jours sont désormais comptés. Les auteurs ne souhaitent pas ici reconstruire les événements révolutionnaires, ne serait-ce que par nécessité de synthèse, mais désirent retourner sur le rôle joué par Internet durant la Révolution.

Ce n'est pas Internet qui a déclenché la Révolution ${ }^{51}$. La Toile - en particulier Facebook - a toutefois joué un rôle non marginal, comme nous l'avons expliqué ci-dessus. C'est à travers Internet que les Tunisiens ont pris connaissance de ce qui était en train de se passer à Sidi Bouzid. Grâce aux téléphones portables, lesquels ont joué un rôle fondamental durant la première phase de la révolte ${ }^{52}$, des photos et des vidéos des événements ont afflué sur la toile. C'est aussi grâce à la toile

45. Touati, 2012.

46. Kuebler, 2011 ; Mihoub, 2011.

47. Chouikha, 2011.

48. Touati, 2012.

49. Lecomte, 2010, 2011.

50. Kallander, 2013.

51. Kuebler, 2011 ; Hibou, 2011 ; Touati, 2012.

52. Chouikha, 2011 ; Kallander, 2013 ; Lecomte, 2011. 
que les médias internationaux ont réussi à couvrir la Révolution. Même Al Jazeera enverra sur les ondes des vidéos trouvées sur Internet ${ }^{53}$.

En plus d'avoir diffusé et véhiculé les informations, Internet et Facebook en particulier ont certainement favorisé et facilité la mobilisation, en tout cas à partir de janvier ${ }^{54}$. L'engagement de nombreux internautes et le travail de beaucoup de bloggers ont sûrement contribué à étendre la contestation envers le régime et à grossir les manifestations ${ }^{55}$.

Le rôle joué par la toile dans les faits révolutionnaires consiste d'un côté, à avoir véhiculé et diffusé l'information, de l'autre, à avoir encouragé, et progressivement facilité, la mobilisation. Cet espace d'Internet (de Facebook en particulier), s'il est circonscrit et précis, est absolument considérable.

\section{L'EXPÉRIENCE DE SFAXONLINE}

\section{Un projet commun}

L'histoire de SfaxOnline est véritablement une histoire de rencontres ; la première est celle entre Yaich et les Sfaxiens de la diaspora.

"Moi j'avais déjà un blog - "Sfax vous aime" - quand je suis entré en contact avec des Sfaxiens émigrés et résidents à l'étranger. C'est à partir de cette rencontre et de l'envie de s'engager ensemble, de faire front commun, que SfaxOnline est né. Nous avons simplement décidé de diriger nos énergie dans un projet commun $»^{56}$.

Le projet commun prend ainsi la forme de SfaxOnline, mis en ligne en 2008 et se présentant dès le départ comme un espace participatif, ce pour quoi un forum est lui aussi activé.

L'objectif des créateurs et des animateurs du site est double: informer les citoyens et les alerter, favorisant de la sorte un réveil de I'esprit critique. Informer est une façon de dénoncer la dégradation croissante de Sfax et de raconter les conditions dans lesquelles la ville se trouve. Ce travail préliminaire d'informations - un récit qui est aussi

53. Lecomte, 2011 ; Touati, 2012.

54. Mihoub, 2011 ; Soussi, 2011 ; Touati, 2012.

55. Mihoub, 2011 ; Lecomte, 2011.

56. Communication personnelle avec Chokri Yaich. 
le fruit d'un travail de recherche et de divulgation - sert à ceux qui écrivent pour solliciter les débats et les confrontations.

"SfaxOnline était conçu pour provoquer des réactions et réveiller les consciences, nous voulions que les protagonistes en soient les citoyens et c'est dans ce but que nous essayions de stimuler une sorte de journalisme citoyen »(ENT07).

Le site se remplit rapidement de nouveaux contenus et un groupe fixe de collaborateurs se met en place. La recherche de personnes capables de traiter certains thèmes et disposées à le faire suit des itinéraires différents. Dans certains cas, on tente d'impliquer des personnalités connues ou amies. Dans d'autres, l'implication passe par le réseau Internet, parfois directement à travers SfaxOnline.

"J'ai été coopté par un ami qui y écrivait. Je suis entré dans le groupe de travail un an après le lancement du site. Je le connaissais déjà, j'avais participé à différents échanges de commentaires dans le forum et je l'appréciais »(ENT02).

Ce qui s'institue alors, c'est un groupe de travail relativement hétérogène, constitué de personnes ayant avant toute chose des compétences et des expériences différentes. L'âge des participants, le degré de participation et d'activisme politique dans le passé diffèrent. Ainsi, des personnes avec des expériences limitées dans ces domaines en côtoient d'autres déjà rodées avec un passé parfois significatif. De fait, tout ce qui apparaît sur le site, même si écrit individuellement, est le résultat d'une grande collaboration, l'expression d'un groupe.

"Chacun mettait son article sur "chantier de rédaction", comme ça on pouvait tous s'en faire une idée, proposer des modifications et rediscuter certains passages, de manière extrêmement démocratique »(ENT04).

Une dimension collective est recherchée et trouvée, le groupe collabore et se coordonne, afin de donner du poids à ce qui est communiqué mais aussi par prudence pragmatique.

"L'objectif était d'éviter la censure " (ENT02).

"Il fallait trouver les formules justes. Plutôt que des attaques frontales à un système qui n'avait rien de démocratique, nous avons trouvé notre propre ligne, qui est devenue la ligne de SfaxOnline : I'information en vue de la confrontation" (ENT01). 
Parfaitement conscient des risques encourus, bien plus graves que la censure elle-même, le groupe parvient à trouver sa ligne. On écrit à partir de faits et de données précis, avec une grande rigueur.

"Ce qui ne pouvait pas être démontré n'était pas écrit, sauf si c'était flagrant. Il fallait écrire sur ce qui était clair et évident, sans avoir besoin d'apporter des preuves, parce que dans de tels cas, qui commande a vite fait de les demander et de les salir " (ENT01).

Un langage simple et direct est choisi afin d'apparaître clair et accessible à tous. Les écrits sont courts, synthétiques et concis, ce qui les rend adaptés à la toile mais aussi plus faciles à être retenus et ainsi reproduits. Cela évite en outre de fournir trop d'éléments sur lesquels on aurait p. éventuellement être attaqués.

"Il s'agissait de savoir écrire de manière générique mais pas vague » (ENT06).

Comme de nombreux internautes tunisiens, avant et après eux, les protagonistes de SfaxOnline ont souvent recours à I'humour et à la satire.

"Dans un système dictatorial, c'est presque physiologique. On ne peut pas parler directement de beaucoup de choses, on doit donc recourir à des métaphores et inventer des histoires avec de l'humour. On l'a fait nous aussi, on a essayé de soulever des problèmes et d'attirer l'attention sur certaines questions sans avoir l'air de vouloir faire de la politique »(ENT07).

\section{Au delà du réseau}

De nombreux lecteurs, simples citoyens et exposants de la société civile, commencent à envoyer au groupe des preuves d'estime. Si le nombre de ceux qui suivent le site et démontrent leur soutien au groupe grandit, c'est aussi parce que le travail de SfaxOnline ne reste pas seulement en ligne et que le groupe de travail commence à interagir en dehors d'internet.

"On se rencontrait souvent, parfois juste pour un café. Notre rapport à nous n'était pas virtuel » (ENT03).

"On était un site mais on a créé une vraie vie associative" (ENT10).

La visibilité du site grandit aussi parce qu'au fil des ans, il épouse différentes causes et essaye, quand cela est possible, de promouvoir des actions concrètes. L'une de celles-ci - signalée par tous les interviewés 
avec une emphase particulière - est certainement la mobilisation autour du cas du Lot 23A. Le lot en question fait partie du projet Sfax El Jadida, qui constitue l'initiative majeure de requalification urbaine dans le centre historique. Il fut lancé dans les années 80 et soutenu par des capitaux saoudiens et koweitiens ${ }^{57}$, ainsi que par l'administration communale, qui fait elle aussi partie de l'actionnariat de la Société d'Aménagement de Sfax El Jadida (SASEJ) gérant le projet. Le travail de vingt années de la Société est, selon les animateurs du site, catastrophique ${ }^{58}$. Dans les plans originaux, dont la Société s'est progressivement éloignée, la superficie totale constructible était fixée à 300000 mètres carrés, alors qu'elle est arrivée à un million ${ }^{59}$. SfaxOnline commence à écrire sur le sujet en $2009^{60}$. Son objectif est d'empêcher la construction du Lot 23 A, aire centrale pas encore cimentée et pour laquelle le projet de départ prévoyait un usage d'intérêt collectif.

La confrontation continue avec différents articles, au cours de 2010, dans une intensité croissante qui voit le site prendre position contre l'incapacité du nouveau conseil communal de s'opposer à la tentative de spéculation ${ }^{61}$. On passe alors rapidement de la dénonciation en ligne aux faits, en essayant de capitaliser l'attention et les consensus attirés grâce au site.

"Nous avons constitué un groupe de pression et nous nous sommes entourés d'hommes de loi pour nous protéger et pour que notre bataille soit plus efficace. Nous avons lancé des pétitions, plusieurs, qui ont été signées par un nombre important de Sfaxiens. Nous avons commencé un véritable bras de fer » (ENT04).

Le bras de fer continue jusqu'aux jours de la Révolution mais ne s'arrête pas avec elle. A deux mois à peine de la chute du régime, le tournant définitif semble se concrétiser.

"On a gagné!

Le Ministère de la Culture a interdit la construction du Lot 23 A !

[...] Ca a été une bataille dure, longue et difficile, qui nous a fait passer par des moments de démoralisation, des moments d'incertitude, on avait même cessé de croire en la justice. [...]

Vive la Révolution !!! » ${ }^{62}$.

57. Megdiche, 2005.

58. SfaxOnline, 2012.

59. Ellouze, 2009a.

60. Voir SfaxOnline, 2009; Ellouze, 2009a, 2009b.

61. Ellouze, 2010.

62. Ellouze, 2011. 
En réalité, avec un parcours pas différent de celui qui marque la difficile transition démocratique du pays, la SASEJ essaye de profiter de la fragilité des institutions locales et de la faible capacité de contrôle du nouveau gouvernement pour relancer les travaux, en contradiction totale avec les lignes ministérielles et les sentences des tribunaux. Encore une fois, le site donne voix au mécontentement de la société civile $^{63}$. Dans les mois suivants, la tentative de construire est à nouveau bloquée; I'hypothèse de réaliser une structure à usage collectif, proposée plusieurs fois par le site, se concrétise.

\section{Résister}

SfaxOnline n'a pas tardé à attirer l'attention des autorités, entre autres choses pour le genre d'initiatives et de positions décrites précédemment.

"Contrôlés, on l'était tous, mais nous on savait que l'Agence tunisienne d'Internet nous suivait parce qu'on avait nos contacts à Tunis qui nous tenait informés » (ENT05).

En outre, ceux qui écrivaient étaient parfaitement conscients des risques et travaillaient d'ailleurs pour combattre la peur des lecteurs potentiels.

"Quand j'ai commencé à écrire les premiers articles sur SfaxOnline, je les signalais à mes contacts par mail ou par sms. Beaucoup de gens m'avouaient que par peur, ils n'osaient pas ouvrir le site. Ils avaient peur d'être détectables et donc signalés et peut-être enlevés par la police. Dans ce cas, on copiait le texte de l'article et on l'envoyait directement par mail. C'est comme ça, entre autres, qu'on a réussi à avoir jusqu'à 25.000 lecteurs, contre les 200 ou 300 initiaux» (ENT08).

La rigueur, la prudence et tous les efforts faits pour rendre les contenus du site inattaquables ne suffisent pas, en effet, à éviter les menaces et les rétorsions.

"J'ai subi de tout, à part de la prison. La prison est la seule chose qui m'ait été épargnée mais pour le reste, on m'a tout fait. J'ai payé ! On m'a coupé le téléphone, abimé la voiture, insulté à plusieurs occasions, on a forcé la porte de mon bureau! C'était le prix à payer» (ENT01).

63. Elloumi, 2012. 
Progressivement, I'hostilité des autorités grandit effectivement, quasiment jusqu'à arriver à un point de rupture.

"En 2010, à la fin de l'année, on était proche de la censure. On a su que l'un d'entre nous aurait été arrêté pour avoir écrit sur le site. L'ordre était parti et ce serait arrivé si la Révolution n'avait pas explosé, qui a été plus rapide heureusement!»(ENT09).

Si ce n'avait pas été pour la Révolution, I'histoire de SfaxOnline et de ses protagonistes aurait probablement pris une autre tournure. En janvier 2011 cependant, comme on le sait, le régime de Ben Alì tombe. Le dictateur chassé, les Tunisiens peuvent enfin s'exprimer sans craindre la répression. Dans ce climat de liberté d'expression inédit, le rôle de SfaxOnline ne peut évidement que changer. D'une façon plus générale, c'est l'espace à l'intérieur duquel évoluait le site qui se transforme.

"La liberté d'expression a changé sensiblement la géographie de l'information » (ENT02).

Le site reste en ligne et continue cependant, avec une régularité moindre, à proposer des contenus originaux, en tout cas dans les premiers mois. La page Facebook reste quant à elle résolument plus active que le site puisqu'en décembre 2013, elle est encore suivie par plus de 57.000 internautes.

Les personnes interviewées rapportent en effet qu'une partie de la mission du site semble s'être éteinte.

"La spécificité de SfaxOnline était d'écrire des articles courageux : avant la Révolution ici, il n'y en avait pas de comme ça. Maintenant il y a la liberté et tout le monde écrit »(ENT03).

Cependant, I'expérience du groupe de SfaxOnline ne finit pas avec la Révolution, elle donne un élan à ses protagonistes qui, même à travers la visibilité acquise avec le site, assument de nouveaux engagements. Le 17 janvier, Mohamed Aloulou est nommé Ministre de la Jeunesse et des Sports. Il le restera jusqu'au $1^{\text {er }}$ juillet 2011.

"Moi après la Révolution je me suis retrouvé Ministre. Pourquoi ? Parce que les choses considérées comme des erreurs avant la Révolution sont devenues des mérites. Ce qui était une honte - disons la contestation - est devenu une qualité, à l'improviste ${ }^{64}$.

64. Communication personnelle avec Mohamed Aloulou. 
Chokri Yaich deviendra député en octobre 2011, comme nous I'avons déjà mentionné. Plusieurs animateurs du site confluent vers d'autres organisations. Pour tout le monde, SfaxOnline se révèle une expérience capable de marquer la personnalité politique.

"L'expérience de SfaxOnline m'a certainement donné du courage et m'a donné envie de participer de manière différente » (ENT08).

Tous les protagonistes conservent la forte sensation d'avoir participé à un projet important, dont le bilan est extrêmement positif.

"L'histoire de SfaxOnline est extraordinaire, absolument unique en son genre en Tunisie. Je suis vraiment fier d'en avoir fait partie »(ENT04).

"On voulait - et on a réussi - créer de l'opinion et faire en sorte que les Tunisiens s'expriment »(ENT05).

"Moi je pense que SfaxOnline a vraiment été au départ du réveil de la société civile de Sfax » (ENT09).

Au-delà de ces parcours individuels, les animateurs du site partagent tous le désir de donner une suite à SfaxOnline. Si la page Facebook n'a pas cessé de proposer et de relancer de nouveaux contenus (pas forcément originaux), le site lui, même s'il n'est pas abandonné, est peu actif en 2013 (depuis décembre, il est même souvent hors service). Le projet de certains animateurs - actuellement en recherche de financement - est de créer, sur la ligne de SfaxOnline, une série de portails Internet (24, soit un par gouvernorat) qui accueillent et promeuvent un journalisme citoyen de proximité.

\section{CONCLUSION}

Malgré l'intérêt insuffisant de la recherche pour ce sujet, Internet a été, pour les Tunisiens résidant dans le pays ou à l'étranger, un espace de confrontation politique et de contestation du régime bien avant la Révolution. SfaxOnline en raconte les antécédents et son histoire recueille divers éléments qui ont caractérisé, à partir de la fin des années 90, les différentes phases de l'utilisation d'Internet en tant qu'instrument de contestation.

L'expérience des blogs est à la racine de SfaxOnline, qui nait sur les traces d'un blog («Sfax je vous aime ») de Chokri Yaich. Ils constituent le contexte au sein duquel, de 2005 à l'affirmation des réseaux sociaux, le monde du numérique tunisien conflue. 
Le rôle joué par les Tunisiens de la diaspora en tant que pionniers de la contestation numérique est important: SfaxOnline nait aussi de la rencontre entre un Sfaxien résidant en Tunisie (Yaich) et ses concitoyens résidant à l'étranger.

La conscience du prix à payer pour la liberté d'expression est élevée: SfaxOnline arrive à éviter la censure, mais ses animateurs subissent menaces et représailles. Les récits des personnes interrogées laissent apparaître le contrôle asphyxiant des autorités tunisiennes et la nécessité de peser chaque mot, ceci afin d'éviter des conséquences bien plus graves que la censure.

Cette expérience met en évidence la reconnaissance du rôle joué par Internet dans un pays où la dictature anéantissait les espaces de contestation mais aussi simplement de confrontation. Alors que les médias se contentaient de reporter les déclarations des autorités, sur SfaxOnline, les internautes reçoivent des informations réelles sur l'état de leur ville et, dans les espaces participatifs, ils peuvent se confronter et discuter sur des sujets d'intérêt commun.

Ce rôle est bien sûr fondamental. De fait, en dehors de Yaich, les interviewés (qui constituent le gros des animateurs du site sur place) avaient une faible connaissance $d^{\prime}$ Internet. II ne s'agissait pas de bloggers mais de simples internautes qui n'étaient pas habitués à écrire en ligne. Pourtant, conscients du potentiel de l'instrument, ils ont appris à utiliser Internet pour diffuser et promouvoir leur travail personnel et celui du groupe, pour faire entendre leur propre voix.

Enfin, le dernier aspect relevé par cette étude, essentiel, concerne le fait que jusqu'à la Révolution, trop d'analyses sur la Tunisie et sur le monde arabe entier véhiculent le préjugé selon lequel les populations de ces pays seraient sans ambition et incapables d'agir politiquement ${ }^{65}$. L'histoire de SfaxOnline contribue à combattre cette perception erronée d'une société civile apathique et homogène, inerte, statique et résignée. C'est une histoire de résistance et de critique, en et hors ligne, dans un pays où les conséquences des actions sur internet dépassaient largement le cadre virtuel.

65. Voir entre autres Touati, 2012. 


\section{BIBLIOGRAPHIE}

AlLAL A., 2010, « Réformes néolibérales, clientélismes et protestations en situation autoritaire. Les mouvements contestataires dans le bassin minier de Gafsa en Tunisie (2008) », Politique africaine, 1, $\mathrm{n}^{\circ} 117$, p. 107-125.

BarHouml S., 2012, "Facebook en Tunisie avant 2011. Vers une transition démocratique », p. 171-184, in Proulx S., Millette M., Heaton L. (éds), Médias sociaux: enjeux pour la communication, Presses de l'Université du Québec.

Bechir Ayari M., Geisser V., 2011, Renaissances Arabes. 7 questions clés sur des révolutions en marche, Paris : Les Editions de l'Atelier, 160 p.

Ben Mhenni L., 2009, 17.03, Tunisia: Remembering Tunisia's First Internet Prisoner, Global Voices, http://globalvoicesonline.org/2009/03/17/ tunisia-remembering-tunisias-first-internet-prisoner/ [consulté la dernière fois le 15.12.2013]

ChOuikHa L., 2011, "Les nouvelles technologies de communication, vecteurs de la "révolution democratique arabe »: le cas tunisien », AWRAQ, n 3, p. 97-106.

ChOuikHa L., Geisser V., 2010, "Retour sur la révolte du bassin minier. Les cinq leçons politiques d'un conflit social inédit ", L'Année du Maghreb, VI, p. 415-426.

Ellouml K., 2012, 13.09, Sur le lot 23A : un chantier hors la loi ?, SfaxOnline, http://www.sfaxonline.com/actualites-sfax-tunisiemonde/1-sfax-region/2296-le-chantier-du-lot-23a-un-projet-horsla-loi [consulté la dernière fois le 30.11.2013]

Ellouze R., 2009a, 04.03, Il était une fois, Sfax... El Jadida : Heurs et malheurs d'une cité, SfaxOnline, http://www.sfaxonline.com/sfaxidees/sfax-opinions/610-il-etait-une-fois-sfax-el-jadida-heurs-etmalheurs-dune-cite [consulté la dernière fois le 30.11.2013]

Ellouze R., 2009b, 07.11, A propos du lot 23A, je dénonce, SfaxOnline, http://www.sfaxonline.com/sfax-idees/sfax-opinions/940-a-proposdu-lot-23a-je-denonce [consulté la dernière fois le 30.11.2013]

Ellouze R., 2010, 01.10, Le Poing Final!, SfaxOnline, http://www. sfaxonline.com/sfax-idees/sfax-opinions/1497-le-poing-final [consulté la dernière fois le 30.11.2013]

Ellouze R., 2011, 17.03, VICTOIRE! Le projet du LOT 23A est arrêté, SfaxOnline, http://www.sfaxonline.com/actualites-sfax-tunisiemonde/1-sfax-region/1747-victoire-le-projet-du-lot-23a-est-arrete [consulté la dernière fois le 30.11.2013]

Filıu J-P., 2011, La Révolution arabe. Dix leçons sur le soulèvement démocratique, Paris : Fayard, 251 p.

Gonzalez-Quijano Y., 2013, "Promesses fallacieuses? Les médias sociaux et les changements politiques arabes ", Annuaire IEMed de la Méditerranée 2013, p. 65-68. 
Hıвou B., 2011, "Tunisie. Économie politique et morale d'un mouvement social », Politique Africaine, n 121, p. 5-22.

Kallander A.A., 2013, "From TUNeZINE to Nhar 3la 3mmar: A Reconsideration of the Role of Bloggers in Tunisia's Revolution ", Arab Media and Society, Issue 17: http://www.arabmediasociety. $\mathrm{com} /$ ?article $=818$ [consulté la dernière fois le 15.12.2013]

Khamis S., 2013, "Le rôle des médias dans les transitions arabes: comment le "cyber-activisme » est en train de bouleverser les panoramas politique et communicationnel », Annuaire IEMed de la Méditerranée 2013, p. 59-64.

Kuebler J., 2011, "Les révolutions arabes et le web 2.0 Tunisie et Égypte ", Revue Averroès, Spécial "Printemps arabe", n 4-5: http:// revueaverroes.com/category/numero-4-5-082011/les-revolutionsarabes-et-le-web-2-0-tunisie-et-egypte/ [consulté la dernière fois le 15.12.2013]

LeCOMte R., 2010, "Internet et la reconfiguration de l'espace public tunisien : le rôle de la diaspora », tic\&société [En ligne], Vol. 3, $\mathrm{n}^{\circ} 1-2$, mis en ligne le 12 janvier 2010 : http://ticetsociete.revues. org/702 [consulté la dernière fois le 15.12.2013]

Lecomte R., 2011, "Révolution tunisienne et Internet: le rôle des médias sociaux ", L'Année du Maghreb, VII, p. 389-418.

Megdiche T., 2005, L'évolution de la division sociale de l'espace à Sfax (Tunisie), Colloque International "Les villes au défi du développement durable: Quelle maîtrise de l'étalement urbain et des ségrégations associées ? », 24-27 novembre 2005, Sfax, Université de Sfax (laboratoire SYFACTE). http://eso-gregum.univlemans.fr/lMG/pdf/megdiche-2.pdf [consulté la dernière fois le 15.12.2013]

Mıноuв S., 2011, "Le cyberactivisme à I'heure de la révolution tunisienne », Archivio Antropologico Mediterraneo on line, anno

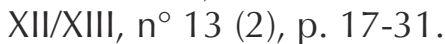

Reporters Sans Frontières, 2011, Prix Reporters sans frontières du Net-citoyen. http://fr.rsf.org/prix-reporters-sans-frontieresdu-14-03-2011,39784.html [consulté la dernière fois le 15.12.2013]

SfaxOnline, 2009, 14.02, Le lot 23A de Sfax El Jadida, SfaxOnline, http://www.sfaxonline.com/sfax-idees/sfax-opinions/586-le-lot-23a [consulté la dernière fois le 30.11.2013]

SfaxOnline, 2012, Dossier Sfax El Jadida http://www.sfaxonline.com/ todo?view=project\&id=38 [consulté le 20.12.2012]

Soussı S., 2011, "Comment faire la révolution à l'heure d'internet? Regard sur le rôle des médias sociaux dans la révolution tunisienne ", Archivio Antropologico Mediterraneo on line, anno XII/XIII, $\mathrm{n}^{\circ} 13$ (2), p. 33-40.

TOUATI Z., 2012, "La révolution tunisienne: interactions entre militantisme de terrain et mobilisation des réseaux sociaux", L'Année du Maghreb, VIII, p. 121-141. 